\title{
Laparoscopic Paediatric Hernia Repair Using Tuohy Needle - Our Preliminary Experience of 100 Cases
}

\author{
Siddhartha Sankar Bhattacharjee ${ }^{1}$, Sneha Daniel², Prasenjit Das ${ }^{3}$, Khorshid Alam Hussain, \\ Nitesh Kumar ${ }^{5}$, Manoj Kumar Singh ${ }^{6}$
}

\begin{abstract}
${ }^{1}$ Department of General Surgery, Silchar Medical College and Hospital, SMC, Silchar, Assam, India. 2Department of General Surgery, Silchar Medical College and Hospital, SMC, Silchar, Assam, India. ${ }^{3}$ Department of General Surgery, Silchar Medical College and Hospital, SMC, Silchar, Assam, India. ${ }^{4}$ Department of General Surgery, Silchar Medical College and Hospital, SMC, Silchar, Assam, India. ${ }^{5}$ Department of General Surgery, Silchar Medical College and Hospital, SMC, Silchar, Assam, India. ${ }^{6}$ Department of General Surgery, Silchar Medical College and Hospital, SMC, Silchar, Assam, India.
\end{abstract}

\section{ABSTRACT}

\section{BACKGROUND}

Congenital inguinal hernia repair is one of the most commonly encountered paediatric surgical procedures. With advancements in minimal access surgery, there is an increasing inclination towards the repair of paediatric inguinal hernia (PIH) laparoscopically over an open procedure. Currently, a single port laparoscopic percutaneous extraperitoneal closure, a simple and reliable method, is being used widely.

\section{METHODS}

100 patients with PIH were treated using a single port LPEC technique from August 2014 to July 2018. The study was conducted with a modified LPEC technique using a Tuohy needle. The deep ring was encircled with the help of Tuohy needle under laparoscopic visualization and a suture was passed through it. The needle was then used to encircle the ring from the opposite side and the initial suture was retrieved with the help of a loop, and a purse string was created to obliterate the deep ring with extracorporeal knotting.

\section{RESULTS}

100 congenital hernias were repaired by LPEC method. Mean age was $5.85 \pm 2$ years . Male to female ratio was $7.3: 1.96 \%$ of cases were unilateral and $4 \%$ were bilateral preoperatively. PPV was found in $8 \%$ of cases intraoperatively. Mean operating time was $22.08 \pm 10.2$ and $27.6 \pm 11.47$ minutes for unilateral and bilateral cases respectively. There were no intraoperative complications and no recurrences. Only one patient developed wound infection.

\section{CONCLUSIONS}

LPEC of hernial sac using a Tuohy needle is a safe and effective method for the repair of PIH. It is easy to perform, faster, and offers good cosmesis with cure for imminent contralateral hernias.

\section{KEY WORDS}

Paediatric Inguinal Hernia, Minimal Access, LPEC, Contralateral Exploration
Corresponding Author:

Sneha Daniel.

R/o Room no. 26, Old PG Hostel,

Silchar Medical College and Hospital, Silchar, Assam, India.

E-mail: snehadaniel1993@gmail.com

DOI: 10.14260/jemds/2020/635

How to Cite This Article:

Bhattacharjee SS, Daniel S, Das P, et al. Laparoscopic paediatric hernia repair using tuohy needle - our preliminary experience of 100 cases. J Evolution Med Dent Sci 2020;9(39):2903-2907, DOI: 10.14260/jemds/2020/635

Submission 22-05-2020,

Peer Review 20-08-2020,

Acceptance 27-08-2020,

Published 28-09-2020.

Copyright (C) 2020 Siddhartha Sankar Bhattacharjee, et al. This is an open access article distributed under Creative Commons Attribution License [Attribution 4.0 International (CC BY 4.0)] 


\section{BACKGROUND}

Congenital inguinal hernia is a common paediatric problem accounting for around $15 \%$ of paediatric surgical cases..$^{1,2}$ This is caused by the failure of closure of the patent process us vaginalis in children with a predisposition for premature neonates. Thus, in most children indirect inguinal hernias enter the abdominal wall laterally to the epigastric vessels at the region of the inner inguinal ring. A variety of approaches have been described for the surgical repair of a paediatric inguinal hernia. However, open hernia repair, herniotomy, is still considered the gold standard with recurrence rates of approximately $1 \%{ }^{2,3}$ The traditional open approach includes an inguinal incision with dissection and high ligation of the hernial sac. But, with advancements in Minimal Access Surgery (MAS), paediatric hernias are being repaired laparoscopically. ${ }^{4}$ Laparoscopy is being used as both a diagnostic tool, and as an approach for repair, including herniorrhaphy. The superiority of either procedure has been much debated upon. Despite higher recurrence rates (up to 5 $\%),{ }^{2}$ several benefits like minimal manipulation of cord structures, better visualization of contralateral hernias, reduced post-operative pain and minimal scarring have also been attributed to the latter procedure.3,5 With gaining experience even recurrence rates have shown a falling trend.

Over time, various techniques of laparoscopic hernia repair have been developed with varying degrees of success. While these techniques all have the ultimate goal of obliterating the PPV, they vary considerably in their approaches to the internal ring (intraperitoneal, extraperitoneal), use of ports (three, two, one), endoscopic instruments (two, one, or none), sutures (absorbable, nonabsorbable), and techniques of knotting (intracorporeal, extracorporeal). ${ }^{2}$

Currently, single port repair and use of extracorporeal knotting is a favoured and reliable technique. Laparoscopic percutaneous extraperitoneal closure (LPEC) is gaining popularity and numerous variations in techniques are being reported.2,6 We used the LPEC technique with a purse-string herniorrhaphy of the sac at the internal inguinal ring using a Tuohy needle and evaluated the outcome of our technique of the repair.

\section{METHODS}

A prospective study was carried out at Silchar Medical College and Hospital for a period of two years (August 2014 - July 2018) following a clearance from the ethical committee. 100 patients aged less than 12 years were operated upon laparoscopically for inguinal hernia repair using a Touhy needle. All cases were done by the first author. A predesigned proforma was used for data collection. Data was collected based on patient demographics, operative details, operative time, post-operative complications, cost and cosmesis. All patients were followed up after operation on 7th day, 1 month, 3 month, 6 month and 1 year and 2 years.

Previous studies have reported an incidence of $6.62 \%$ in male children from birth to 15 years of age. Based on the same, the sample size was calculated using the formula

$$
\mathrm{N}=\mathrm{z}^{2} \times(\mathrm{p} \times \mathrm{q}) / \mathrm{d}^{2}
$$

Where, $\mathrm{n}=$ sample size, $\mathrm{p}=$ prevalence, $\mathrm{q}=1-\mathrm{p}, \mathrm{d}=$ margin of error $7 \%, z=1.96$ at $95 \% \mathrm{CI}$. Using the calculation, the sample size calculated was 50 . To increase the power of the study, we included double the minimum sample size required, and took a sample of 100 .

\section{Surgical Technique}

Under general anaesthesia and appropriate muscle relaxation, a pneumoperitoneum with $\mathrm{CO}_{2}$ at a pressure of 8-10 $\mathrm{mm}$ of $\mathrm{Hg}$ was created using a Veress needle through an umbilical incision. A $10 \mathrm{~mm}$ transumbilical port was placed and a $10 \mathrm{~mm}$ $30^{\circ}$ scope was introduced into the abdomen. Patient was placed in Trendelenburg position and both the hernial rings were inspected for defects (Picture 1). After the first 10 cases, we shifted to a $5 \mathrm{~mm}$ scope when it became available.

A $2 \mathrm{~mm}$ stab wound was made at the deep ring. An $18 \mathrm{G}$ Tuohy epidural needle was used to guide a purse string suture around the deep ring. The anterior one third of the needle was curved which allowed negotiation around the hernial ring with greater ease. The needle was guided along the lateral border of the ring in the preperitoneal space. It was advanced just medial to the gonadal vessels and the vas deferens and was intraperitonealised (Picture 2). A 3-0 Prolene suture was guided through the barrel of needle to reach the peritoneal cavity and the needle was withdrawn keeping the suture inside (picture 3). A loop was created using Prolene inside the barrel of the Tuohy needle in a manner that it lay just inside the tip of the needle only coming out when pushed from the outside. The needle was guided from the medial border of the ring through the same stab wound and was advanced in the preperitoneal space just medial to the gonadal structures and was then intraperitonealised through the earlier puncture (Picture 4). The suture was pushed from the outside such that the loop opened up at the tip of the needle. The free suture which was left inside was captured in the loop (picture 5). The loop was then pulled from the outside so that it entered the barrel of the needle along with the captured free suture. 4 out of 10 times during the first 10 cases, an additional $3 \mathrm{~mm}$ grasper was required for capturing the free end of the suture inside the loop. The grasper was introduced below and lateral to the umbilicus ipsilaterally, without any port. Later with experience, we were able to do the repair without additional instrumentation or ports.

Finally, the needle along with the loop was pulled to retrieve the free end of the Prolene suture which now encircled the deep ring completely (picture 6). It was tied extracorporeally (6 knots) and cut. The ring was completely obliterated as seen from inside (picture 7). Skin was lifted such that the knot retracted inside the skin.

The contralateral hernial ring was inspected to rule out a patent processus vaginalis (PPV) and if found, was repaired using the same technique even in the absence of clinical symptoms considering them as potential hernias. In case of females the needle was intraperitonealised through the same peritoneal defect each time as there was no risk of injuring any vital structures. The fascia of umbilical port was closed using 3-0 Vicryl and skin with 3-0 nylon. The $3 \mathrm{~mm}$ wound was closed with 3-0 nylon. The $2 \mathrm{~mm}$ groin needle stab incision was not sutured. 

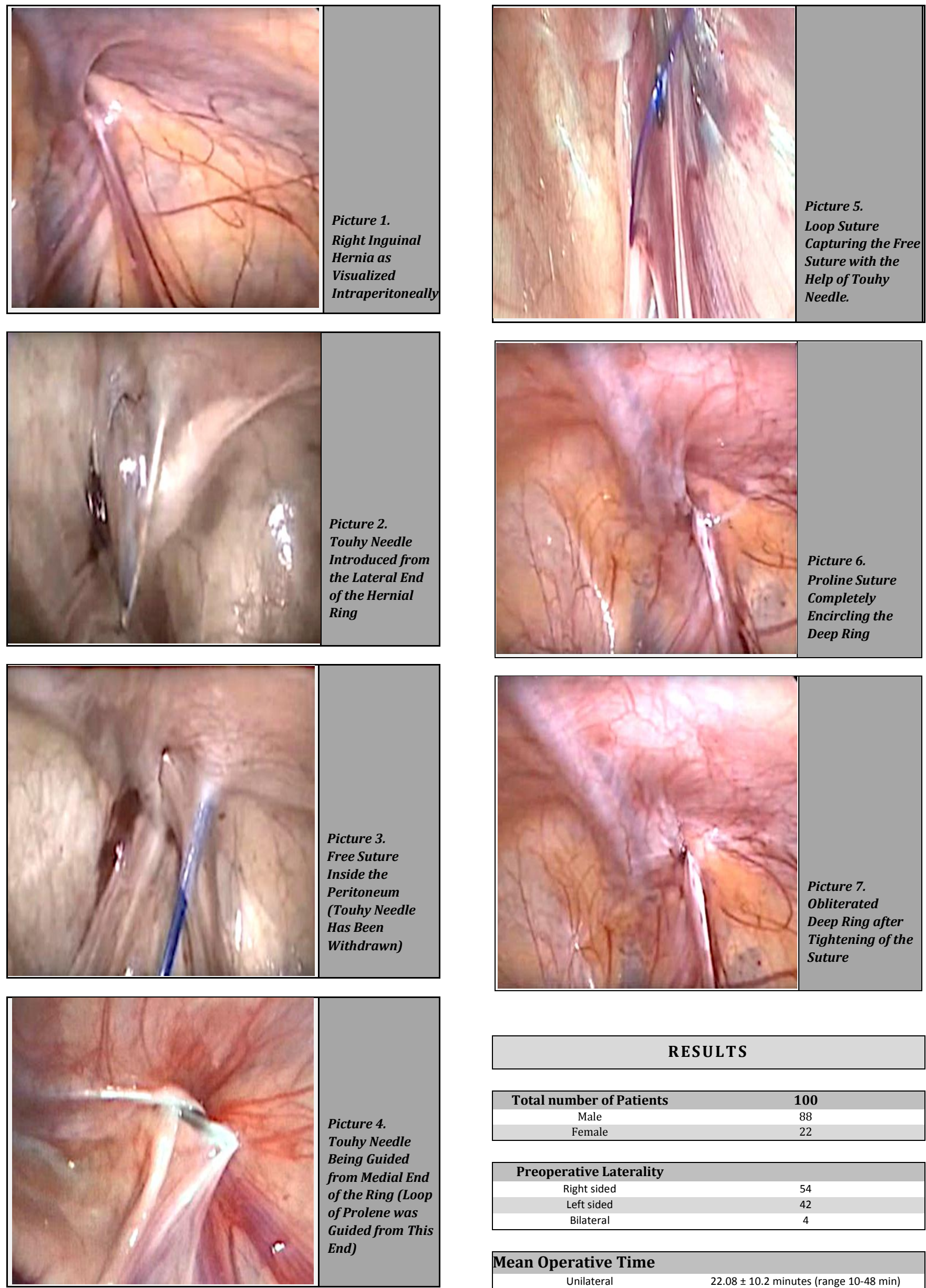

\section{RESULTS}

\begin{tabular}{|cc|}
\hline Total number of Patients & $\mathbf{1 0 0}$ \\
Male & 88 \\
Female & 22 \\
\hline
\end{tabular}

\begin{tabular}{|cc|}
\hline Preoperative Laterality & \\
Right sided & 54 \\
Left sided & 42 \\
Bilateral & 4 \\
\hline
\end{tabular}

\begin{tabular}{|c|c|}
\hline \multicolumn{2}{|c|}{ Mean Operative Time } \\
\hline Unilateral & $22.08 \pm 10.2$ minutes (range $10-48 \mathrm{~min}$ ) \\
\hline Bilateral & $27.6 \pm 11.47$ minutes (range $15-50 \mathrm{~min}$ ) \\
\hline
\end{tabular}


During the two year study period between August 2014 to July 2018, 100 patients underwent laparoscopic inguinal hernia repair. Of them, 88 cases were males and the rest females. M: F ratio was 7.3:1. The mean age of the patients was $5.85 \pm 2$ years, ranging between 3 yrs. to $10 \mathrm{yrs}$. Preoperatively $96(96 \%)$ cases were unilateral and 4 (4\%) cases were bilateral. Among unilateral hernias, right sided hernias were repaired in 54 cases and left sided in 42 cases. Intraoperatively, a patent processus vaginalis (PPV) was found contralaterally in $8(8 \%)$ of cases. A contralateral PPV was considered as a defect requiring repair if the air insufflations demonstrated a hernia in the groin or the opening extended beyond the deep ring. Therefore, bilateral repair was done finally in $10(10 \%)$ of cases. Mean operative time for unilateral repairs was $22.08 \pm 10.2$ minutes (range 10-48 $\mathrm{min}$ ) and for bilateral repair was $27.6 \pm 11.47$ minutes (range $15-50 \mathrm{~min}$ ). There was significant reduction in operative time with our experience.

All patients were followed up to minimum of two years, 15 patients were followed up for 3 years and 24 for 2.5 years. There were no intraoperative complications in any of the cases. In the post-operative period, one patient developed port site infection which was managed with oral antibiotics. No hematomas, recurrence, testicular atrophy, hydrocoele, suture granulomas or umbilical hernia were noted in any of the cases.

\section{DISCUSSION}

Laparoscopic repair of congenital inguinal hernia is being taken up by many surgeons in an endeavour to reduce pain and achieve better cosmesis. The collateral damage is also minimal due to enhanced visualization and magnification provided by the advanced laparoscopic instruments and technologies with the additional benefit of contralateral groin exploration. ${ }^{2}$ the laparoscopic approach also avoids access injury to the cord structures and vessels, decreases operative time and enables easy identification of unsuspected direct or femoral hernia. It has also been reported to prevent metachronal hernias and excessive scar formation while providing a chance at simultaneous repair of contralateral PPVs or hernia. Two MAS techniques are commonly used for congenital hernia repair; first being a multiport approach using intracorporeal suturing, and the second being a transcutaneous approach using a simpler extracorporeal suturing technique. Intracorporeal suturing has a steep learning curve, requires more ports and is tedious. ${ }^{2}$ Laparoscopic percutaneous extraperitoneal closure (LPEC), a simplified laparoscopic herniorrhaphy procedure is gaining popularity with many a surgeons and it is easier to perform for the less experienced. Initial concerns about the high recurrence rates are now believed to be caused by premature absorption of the suture materials before stable scar formation at the level of the internal inguinal ring took place. Introduction of nonabsorbable suture materials has thus seen a marked diminution in the recurrence rates. Resection or dissection of the hernial sac have also reported to reduce the incidence of recurrent hernias. Many modifications and different techniques of the LPEC have been reported, with their own merits and demerits.6,7 LPEC is also associated with reduced operative time, lower recurrence rates and better cosmesis than intracorporeal suturing. $2,6-7$
Single port LPEC is becoming the procedure of choice for most surgeons. But the special instruments used are not easy to obtain and many procedures employ a two puncture technique. Different needles and instruments have been used by different surgeons for encircling the deep inguinal ring like Lapaher closure, ${ }^{8}$ the Reverdin Needle, ${ }^{9}$ an $18 \mathrm{~F}$ vascular catheter and a hooked $\operatorname{pin}^{10}$ and $2-0$ suture on a $36-\mathrm{mm}$ needle. ${ }^{11} \mathrm{Li}$ et al. used a Tuohy needle to guide the suture around the deep ring after hydrodissection and the epidural catheter as a suture catching device for the herniorrhaphy. McClain et al. also used Tuohy needle but he used a looped suture through the needle as a capturing device without any hydrodissection. ${ }^{12}$

Our technique differed from McClain's as we first introduced the free suture from the lateral end of the ring and captured it from the looped suture from the medial end of the ring. There is a chance of slippage of the free suture from the loop without the needle and leading to the repetition of the entire procedure as happened when we applied this technique before initiation of the study. In our method, the free suture was secured with the loop inside the barrel of Tuohy needle and the chance of slippage was minimal. It also avoided the need for an additional grasper to catch the free suture end as the loop could be manipulated by the needle. It was convenient for us to create the loop inside the needle as it aided the smooth passage of the needle. No hydrodissection was performed as we were satisfactorily able to negotiate around the vas and vessels due to curved blunt tip of the Tuohy needle.

Initially, some difficulty was encountered (4 cases) in capturing the free suture in the loop and an extra grasper was used. But with experience, all manoeuvres were successfully done with a single port. We didn't find any large rings requiring the use of extra ports.

Many single port techniques used two puncture technique, 2,8-11 which may lead to incorporation of some subcutaneous tissues, nerves and muscles in the knot resulting in loosening of the knot causing subsequent recurrence.6,13,14 The tip of the needle was kept under constant visualization to avoid any extra tissues and all manoeuvres were performed through a single puncture.

Only one patient developed wound infection as a complication in our study which was managed with oral antibiotics only. There were no recurrences, hydrocoele or atrophy of the test is proving the safety and high efficacy of the procedure. Li et al. ${ }^{6}$ reported a recurrence of $0.48 \%$ in 251 cases and McClain et al. ${ }^{12} 0.56 \%$ in 502 cases which were also minimal.

The complex laparoscopic skills like intracorporeal suturing, additional ports and manipulation are not required which expedites the procedure, making it easy to learn. The skill can be imparted to beginners in laparoscopic surgery after they attain some minimal skills and even to junior doctors. The procedure is cost effective with excellent cosmetic results.

\section{CONCLUSIONS}

Increasing expertise has presented laparoscopic repair as a viable option in paediatric hernia repair. With similar recurrence rates, better cosmesis and minimal risk of injury to 
the cord structures due to better visualisation, it is a superior alternative to open techniques. While various approaches and modifications have been tried with varying degrees of success, the aim remains to cut short the learning curve while making the process cost effective. Our experience in the preliminary 100 cases with LPEC using a Touhy needle using our technique proved to be a very safe and cost-effective method of paediatric hernia repair. It is less complex, has a faster learning curve, and has a better prognosis.

Financial or Other Competing Interests: None.

\section{REFERENCES}

[1] Lloyd DA, Rintala RJ. Inguinal hernia and hydrocele. In: O'Neill JA, Rowe MI, Grosfeld JC, et al, eds. Paediatric surgery. St. Louis: Mosby 1998:1071-86.

[2] Bharathi RS, Arora M, Baskaran V. Minimal access surgery of paediatric inguinal hernias: a review. Surg Endosc 2008;22(8):1751-62.

[3] Kastenberg Z, Bruzoni M, Dutta S. A modification of the laparoscopic transcutaneous inguinal hernia repair to achieve transfixation ligature of the hernia sac. Journal of Paediatric Surgery 2011;46(8):1658-64.

[4] Shalaby R, Ismail M, Dorgham A, et al. Laparoscopic hernia repair in infancy and childhood: evaluation of 2 different techniques. Journal of Paediatric Surgery 2010;45(11):2210-6.

[5] Dutta S, Craig A. Transcutaneous laparoscopic hernia repair in children: a prospective review of 275 hernia repairs with minimum 2-year follow-up. Surg Endosc 2009;23(1):103-7.
[6] Li S, Li M, Wong KKY, et al. Laparoscopically assisted simple suturing obliteration (LASSO) of the internal ring using an epidural needle: a handy single-port laparoscopic herniorrhaphy in children. J Paediatr Surg 2014;49(12):1818-20.

[7] Chang YT. Technical refinements in single-port laparoscopic surgery of inguinal hernia in infants and children. Diagnostic and Therapeutic Endoscopy 2010;2010:392847.

[8] Oue T, Kubota A, Okuyama H, et al. Laparoscopic percutaneous extraperitoneal closure (LPEC) method for the exploration and treatment of inguinal hernia in girls. Paediatr Surg Int 2005;21(12):964-8.

[9] Shalaby RY, Fawy M, Soliman SM, et al. A new simplified technique for needlescopic inguinal herniorrhaphy in children. J Paediatr Surg 2006;41(4):863-7.

[10] Chang YT, Lee JY, Tsai CJ, et al. Preliminary experience of one-trocar laparoscopic herniorrhaphy in infants and children. J Laparoendosc Adv Surg Tech A 2011;21(3):277-82.

[11] Kumar A, Ramakrishnan TS. Single port laparoscopic repair of paediatric inguinal hernias: our experience at a secondary care centre. J Minim Access Surg 2013;9(1):712.

[12] McClain L, Streck C, Lesher A, et al. Laparoscopic needleassisted inguinal hernia repair in 495 children. Surg Endosc 2015;29(4):781-6.

[13] Bharathi, RS, Arora M, Baskaran V. How we "SEAL" internal ring in paediatric inguinal hernias. Surg Laparosc Endosc Percutan Tech 2008;18(2):192-4.

[14] Chan KL, Chan HY, Tam PKH. Towards a near-zero recurrence rate in laparoscopic inguinal hernia repair for paediatric patients of all ages. Journal of Paediatr Surg 2007;42(12):1993-7. 